
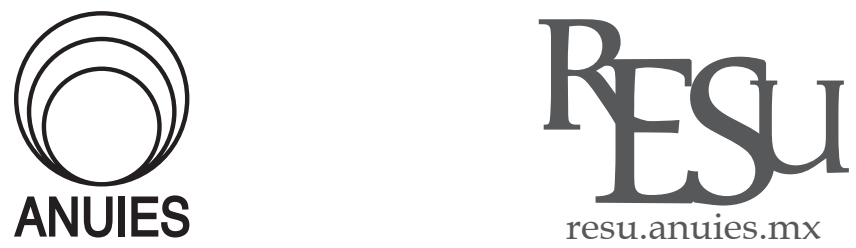

ARTí́cULO

\title{
La apropiación de la política educativa en las universidades públicas mexicanas: entre la ambigüedad y la diversidad
}

\author{
The appropriation of educational policy in Mexican public universities: \\ between ambiguity and diversity
}

\author{
Iván Alejandro Salas Durazo*, Angélica Buendía Espinosa** y Ana Beatriz Pérez Díaz ${ }^{* * *}$ \\ Financiamiento: El presente escrito se realizó con financiamiento del fondo SEP-CONACYT, convenio no. 131978, "Efectos \\ de las políticas gubernamentales en las Universidades Públicas Mexicanas (1989-2009): análisis comparativo de \\ su diversidad y complejidad" \\ *Universidad de Guadalajara. Zapopan, México Correo electrónico ivan.salas.durazo@gmail.com \\ **Universidad Autónoma Metropolitana. Ciudad de México, México. \\ ***Universidad Autónoma Metropolitana. Ciudad de México, México.
}

Recibido el 20 de enero del 2018; aceptado el 25 de junio del 2019

\section{Resumen}

En este artículo se valora el grado de apropiación de la política de educación superior en cuarenta Universidades Públicas Mexicanas (UPM). Se analiza la relación entre indicadores institucionales y escenarios deseables planteados en los principales programas públicos asociados al campo de la educación superior, a partir del cálculo de índices multidimensionales basados en conjuntos de lógica difusa. Como principales hallazgos se identifica la ambigüedad de la política para determinar su alcance en realidades diferenciadas; asimismo, la configuración actual fortalece la competencia y el desarrollo de vocaciones institucionales dando como resultado una ampliación de las brechas en el desempeño institucional.

Palabras clave: Políticas públicas; Universidades públicas; Análisis multidimensional; Diversidad. 


\begin{abstract}
In this paper, the degree of appropriation of the higher education policy in forty Mexican Public Universities (UPM) is evaluated. In such a manner, the relationship between institutional indicators and the desirable scenarios proposed in the main public programs associated to the field of higher education through the calculation of multidimensional indexes based on fuzzy logic is analyzed. Main findings show the policy ambiguity to determine its scope in differentiated realities; it also reinforces competition and the development of institutional abilities, resulting as an expansion of fractures in the institutional performance.
\end{abstract}

Keywords: Public policy; Public universities; Multidimensional analysis; Diversity.

\title{
Introducción
}

$\mathrm{P}$ or más de dos décadas el interés de la política pública en el campo de la educación superior en México se ha centrado en fortalecer la calidad educativa a través de la evaluación de las universidades públicas. La competencia por el acceso a recursos económicos - tendencialmente más escasos y cada vez etiquetados de manera más específica - se ha convertido en una máxima en las estrategias de cada institución. Hemos atestiguado cómo algunas universidades han respondido de manera más favorable a las exigencias de la política posicionándose como ejemplos de calidad, excelencia y eficiencia; complementariamente está presente otro grupo que, si bien participa en los programas, dada la relación evaluaciónfinanciamiento, muestra mucho menor dinamismo. Tales asimetrías son analizadas mediante dos cuestionamientos: ¿cuáles son los factores que inciden en la capacidad de respuesta de las Universidades Públicas Mexicanas (UPM) a los requerimientos de la política? y ¿cómo medir la capacidad de respuesta en términos de la apropiación de la política educativa?

En este artículo se discute el grado de apropiación de la política de educación superior en cuarenta universidades públicas mexicanas. Para ello se proponen dos índices considerando aspectos asociables tanto a la calidad de la docencia como de la investigación, con base en los principios metodológicos de la lógica difusa y vía los parámetros de desempeño planteados en los principales programas que integran la política educativa para el nivel superior.

Se parte del supuesto de que el proceso de modernización para la educación superior generó desempeños diferenciados entre las UPM, lo cual puede explicarse - entre otros factores - a partir de su propio contexto local y 
regional. De manera que aspectos tales como la dimensión, la diversidad y la complejidad, tanto del sistema de educación superior como del sistema universitario mexicano, encuentran cada vez mayores tensiones en su operación y en sus resultados, considerando el esquema actual de implementación homogéneo que caracteriza al sistema mexicano.

El trabajo está organizado en cinco apartados. En el primero se describen los rasgos principales de la política de modernización para la educación superior, cuyo punto de inflexión se ubica en el Programa para la Modernización de la Educación implementado durante el gobierno de Carlos Salinas de Gortari. En segundo lugar se discuten algunas nociones sobre la diversidad en los sistemas de educación superior y la forma en la que ésta puede ser incorporada en la conducción de la política pública. En tercera instancia se describe el proceso metodológico utilizado para la definición y la construcción de los índices multidimensionales. En la cuarta sección se realiza una valoración relativa sobre el grado de apropiación a partir del valor de los índices. Finalmente se incluye un apartado con las conclusiones e implicaciones más relevantes.

\section{Elementos clave en la política de educación superior en México}

A finales de la década de 1980 México inició una transformación fundamental de la educación superior, la cual se enfocó en modificar el perfil de la institución universitaria tradicional. El Programa para la Modernización Educativa (1989-1994), continuado en sus términos fundamentales por el Programa de Desarrollo Educativo (1995-2000), se caracterizó por redefinir las relaciones entre las agencias gubernamentales, las instituciones universitarias y la sociedad (Ibarra, 2001). Se trata de un punto clave en la historia de la educación superior mexicana que implicó una nueva forma de conducción y regulación de las instituciones, y que ha mostrado continuidad hasta el presente. Las políticas de modernización se sustentaron en la premisa de que el impulso de procesos de evaluación implicaría un mejoramiento en la calidad educativa. La transformación más significativa en estos años fue la institucionalización de un modelo de regulación, conducción y coordinación gubernamental de la educación superior, consistente en la articulación de procedimientos de evaluación asociados a programas de financiamiento extraordinario a concurso bajo el establecimiento de compromisos explícitos de cambio de las instituciones que podrían ser constatados en etapas subsecuentes (Buendía, 2014). 
Entre 1989 y 2001, la evaluación como instrumento por excelencia para el mejoramiento de la calidad de la educación superior se constituyó en el eje transversal de las políticas públicas impulsadas desde finales de la década de 1980. La complejidad, la duplicidad de funciones y los procesos son elementos característicos del diseño institucional para operar los mecanismos de evaluación, de acreditación y de certificación de los procedimientos, actores e instituciones, involucrando a autoridades educativas, organismos no gubernamentales, funcionarios, profesores, estudiantes y egresados. Sin embargo, no es posible hablar de la aspiración gubernamental, es decir, de la operación del Sistema Nacional de Evaluación y Acreditación de la Educación Superior, pues las dinámicas institucionales no se cambian por mandatos legislativos o procesos institucionalizados que arraigan viejas comportamientos y prácticas de los actores implicados.

A lo largo de las tres últimas décadas, el gobierno ha intentado revertir la baja calidad de la educación superior universitaria y tecnológica provocada por su expansión no regulada en la década de los setenta y principios de los ochenta. La intención ha sido romper con la lógica burocrática y la politización de las instituciones universitarias, aumentar el nivel académico de sus programas, así como mejorar la escasa relación que mantenían con la economía y la sociedad. Las políticas han puesto mayor énfasis en la actualización de los planes y programas de estudio y los métodos de enseñanza-aprendizaje; además, se ha apostado por la movilidad estudiantil y los programas de tutoría y de asesoría para facilitar el tránsito de los estudiantes por la universidad. La habilitación y la actualización del personal académico también han sido objeto de programas de gobierno e institucionales a fin de propiciar su desarrollo. Al personal académico se le ha considerado fuente principal de mejora de la calidad en las políticas educativas (Buendía et al., 2017).

Para la investigación se han impulsado políticas y programas que buscan mejorar la vinculación entre la investigación y las necesidades de desarrollo de la planta productiva nacional, así como promover el desarrollo de programas de posgrado en las áreas tecnológicas. Por último, se ha buscado mejorar la infraestructura y asignar recursos para su mantenimiento y apoyo, a la vez de incrementar la inversión en bibliotecas, hemerotecas y sistemas de documentación e información.

Si bien los actuales esquemas y modelos de evaluación, de certificación y de acreditación para las Instituciones de Educación Superior (IES) y para el sistema mismo, han traído efectos positivos, es urgente enfrentar las distorsiones que han provocado debido a su excesivo formalismo, apego desmedido a indicadores de orden cuantitativo y la orientación a los productos en lugar de los procesos, tal como lo muestran investigaciones previas (Buen- 
día et al., 2017; Buendía, 2014; Montaño, 2012; Díaz, 2008; Chehaibar, Díaz y Mendoza, 2007; Ibarra, 2001).

No obstante, los últimos dos programas sectoriales poco han aportado al cambio en las políticas para la educación superior. Por el contrario, se ha asumido que sus efectos son positivos dado el comportamiento, también positivo e incremental, de los indicadores diseñados para la medición de su eficacia. Una evaluación reciente del Programa Fortalecimiento de la Calidad Educativa, realizada por la SEP (2016) y que incluye a la educación superior, concluye que su diseño es adecuado y contribuye a lograr la calidad educativa, resultado sumamente cuestionable, pero que en la práctica es considerado para la continuidad de las políticas. Sin embargo, problemas antiguos y recientemente visibilizados como las pensiones y los compromisos signados con los sindicatos en tiempos de bonanza, hoy asociados a la quiebra financiera de algunas universidades, parecen no vincularse en absoluto con las políticas.

Las UPM han caminado un largo trecho para contender con las políticas públicas que se propusieron reorientar su rumbo. A casi tres décadas del comienzo de la llamada era de la modernización educativa, urge hacer un alto para repensar las políticas en términos de su posible cambio o continuidad. Se trata de un punto de inflexión necesario en el contexto de sus resultados no sólo a nivel de los indicadores, sino también a partir de la investigación educativa que las interpelan.

\section{Diversidad institucional y política educativa: ¿ten- siones irreconciliables?}

En el análisis de la política pública para la educación superior en México, la inherente diversidad del sistema resulta significativa para comprender los distintos efectos y alcances que ha tenido, acorde a las circunstancias históricas, geográficas y políticas de cada institución. Repensar la política requiere su reconocimiento con miras a que su diseño, su implementación y su evaluación se realicen en condiciones más justas, equitativas y acorde a contextos y realidades diferenciadas.

El debate sobre la diversidad se originó de la expansión de los sistemas de educación superior después de la segunda guerra mundial (Meek, Goedegebuure y Huisman, 2000). En Europa recobró importancia entre 1960 y 1979 (Teichler, 2008). En México, fue hacia el año 2002 cuando la ANUIES estableció una tipología para caracterizar a las UPM. Además de reflejar la diversidad institucional del sistema, sirvió como fundamento para el desa- 
rrollo de políticas y programas orientados al mejoramiento de la calidad, el financiamiento y la generación de indicadores de desempeño para efecto de los procesos de evaluación y de acreditación (Fresán y Taborga, 2000).

Trow (1995) conceptualiza la diversidad como las diferencias en la misión, la instrucción y las formas de organización de las instituciones o grupos de instituciones, así como sus regulaciones normativas y relaciones con el gobierno. Huisman, Meek y Wood (2007) la asumen como el número de perfiles organizacionales en el sistema de educación superior y la dispersión de las instituciones en dicho sistema. La diversidad también se observa en la historia organizacional, organización académica y administrativa, estructura curricular o de programas académicos, modelos educativos, perfiles de estudiantes a los que se atiende, así como reputación y prestigio de la universidad en los contextos local, regional y nacional, y los valores que guían la conducta universitaria (Birbaum, 1983).

La diversidad en los sistemas de educación superior tiene tantos efectos positivos como resultados no esperados, e inclusive no deseados. Los primeros permiten identificar necesidades de los estudiantes y del mercado laboral, estimular la movilidad social, asegurar la inclusión política de los diversos grupos de interés, propiciar la “combinación" entre los sistemas masificados y de elite, incrementar el nivel de efectividad en las instituciones, aprovechar los procesos de innovación y lograr un desempeño positivo de los sistemas de educación superior (Teichler, 2008; Vught, 2007; Birnbaum, 1983; Stadtman, 1980; Trow, 1970).

Los efectos no esperados muestran la ampliación en las brechas de desigualdad en el acceso a los recursos en contraste con la tendencia homogeneizadora de la política para orientar la conducción de las universidades. Para Neave (2000) "[...] la diversidad institucional puede conducir a la intensificación de las diferencias interinstitucionales, principalmente en términos de los recursos asignados [...]", es decir, a la polarización de grupos de instituciones más o menos favorecidos, según sea el caso (Ibarra y Buendía, 2013). La continua competencia por los recursos ha ocasionado procesos de mimetización entre las universidades en la búsqueda por igualar a aquellas que se convierten en referentes, principalmente en lo que a investigación corresponde (Zha, 2009; Meek, Goedegebuure y Huisman, 2000).

Por otro lado, la política pública refiere a todas aquellas respuestas que el Estado a través de la toma de decisiones y un proceso de análisis atiende las demandas de la sociedad en forma de normas, programas, instituciones, prestaciones, bienes públicos o servicios. Aguilar (1993) destaca las dificultades que atraviesa el proceso de implementación en la coordinación e integración de las diferencias de los actores a quienes va dirigida. 
En particular, la coordinación de los sistemas de educación superior es compleja, dada la especificidad de las IES, definidas como anarquías organizadas o sistemas flojamente acoplados y caracterizadas por la indeterminación de sus objetivos, así como su fragmentación interna y externa, sus dinámicas y la naturaleza de sus actores (Weick, 1976; Cohen y March, 1974). Analíticamente, Clark (1986) evidencia dicha complejidad al incorporar tres mecanismos de coordinación sistémica: Estado, mercado y la oligarquía académica como tipos ideales de gobernanza en educación superior.

Sin embargo, los cambios que enfrenta este subsistema revelan limitaciones explicativas de los tipos ideales pues desde hace varios años la educación superior a nivel mundial ha cambiado tanto conceptual como operativamente. Entre dichas transformaciones podemos destacar la expansión de la matrícula, la diversificación disciplinar, un mayor dinamismo en el proceso de generación del conocimiento y las modificaciones en la estructura organizativa universitaria asociadas a nuevas funciones de administración y de gestión.

Estos procesos implican la reinvención del vínculo universidad y sociedad, ya que sus problemas y necesidades figuran actualmente en las agendas universitarias impactando directamente en los niveles de exigencia de la enseñanza y la investigación. Esta dinámica ha conducido a una nueva forma de gobierno en la educación superior (Badillo, Buendía y Krucken, 2015; Capano, 2011; Frank y Meyer, 2007). Capano (2011) identifica que un mecanismo de adaptación de las ies ha sido la respuesta racional a las exigencias de la política, a través de la implementación de estrategias que equilibran el apoyo gubernamental y los recursos propios partiendo de un contexto socioeconómico específico.

Tales cambios obligan a repensar la conducción de la política con miras a incorporar la diversidad como una variable significativa para su diseño, su implementación y su evaluación. Un acercamiento teórico a este proceso constata la dificultad para determinar los factores o condiciones que facilitan el éxito de la implementación de la política debido a su relación con los contextos político, económico y social (Cerna, 2013). De igual forma, el tamaño y la complejidad institucionales intervienen en la manera en la que actores en turno responden a las exigencias de la política (McLaughlin, 1987).

En México, las UPM han tenido desempeños diferenciados derivados de su participación en los programas de política. En parte esto puede ser resultado de la falta de coordinación y regulación del sistema derivado de su enfoque federalista-centralista (Rodríguez, 2017). Es necesario reflexionar respecto a las condiciones de gobernabilidad y de gobernanza del sistema, con el reconocimiento de su diferenciación, de su complejidad, de su heterogeneidad y de cómo las instituciones han respondido a su entorno. El propio Rodríguez 
(2017) refiere considerar la posibilidad de avanzar hacia sistemas estatales de educación superior con capacidades propias de gestión en conjunto con la cooperación con el gobierno federal. En este contexto valdría cuestionarse ¿cómo la política educativa puede incorporar la diversidad?, y más aún, ¿cómo hacerlo sin profundizar las brechas de desigualdad?

\section{La multidimensionalidad como herramienta metodológica para el análisis social}

El desarrollo de la capacidad de cómputo y análisis de datos ha transformado las maneras de cuantificar y de entender la realidad social. Cada vez es más frecuente la apropiación de métodos y herramientas provenientes de otras áreas del conocimiento para profundizar en las implicaciones analíticas de fenómenos complejos. Bajo esta óptica el QCA (Análisis Cualitativo Comparado) busca realizar una genuina mediación entre las perspectivas cuantitativa y cualitativa a partir de su combinación.

Ragin (2006) señala que una de las principales debilidades de los métodos cuantitativos tradicionales radica en la búsqueda - casi exclusiva - de efectos netos; es decir, del peso específico de cada factor para explicar un fenómeno. Esto implica la correcta especificación de los modelos para que en conjunto con una base teórica se identifiquen las variables determinantes. Sin embargo, la complejidad y la incertidumbre inherentes al estudio social comúnmente conduce a que dos o más elementos relevantes estén correlacionados entre sí, haciendo imposible en la práctica separar el efecto que tiene cada uno en la explicación.

En ese sentido, el QCA buscar aprovechar la ambigüedad y la incertidumbre como aspectos centrales para el análisis. A diferencia de los métodos hegemónicos, esta aproximación busca explicar un fenómeno a partir de la combinación de factores empleando como base la lógica booleana (Ragin, 2014; Yamasaki y Spreitzer, 2006). Asimismo emplea como base la lógica difusa que, a diferencia de la lógica tradicional - dicotómica y mutuamente excluyente-, posibilita desaparecer las fronteras entre clases a través del establecimiento de grados de pertenencia múltiple (Flores y Luhrs, 2013). De igual forma, la combinación de funciones de pertenencia con las tablas de verdad permite diseñar posibles escenarios y comportamientos fundamentados en la integración de conceptos, teorías, tendencias, datos y testimonios de expertos. A esto se le denomina conocimiento sustantivo.

Esto resulta particularmente útil para el análisis y la evaluación de políticas públicas ya que buscan establecer una relación de causa y efecto. Varone, 
Rihoux y Marx (2006) señalan que la valoración del grado de efectividad de los programas públicos no puede ser atribuida a un insumo en particular, por el contrario se debe conceptualizar como una combinación de factores que en conjunto conducen al éxito o el fracaso de una acción gubernamental determinada.

Para efectos del presente escrito se emplea este método en la construcción de índices multidimensionales y con ello estimar el grado de apropiación de la política educativa de nivel superior por parte de las UPM. Al respecto, Salas y Flores (2017) demuestran para el caso del estudio de la calidad del empleo que esta aproximación soporta la incorporación de un mayor número de variables sin sacrificar la capacidad explicativa con respecto a los índices sintéticos tradicionales; además permite proporcionar explicaciones parciales y globales a partir de un diseño basado en índices y subíndices. Complementariamente, García, Flores y Venegas (2016) identifican dos elementos que en potencia dificultan la construcción de índices multidimensionales: ambigüedad conceptual y la calidad de la información de origen. La primera se refiere a que la conceptualización en el marco de la realidad social no siempre está fundamentada en hechos objetivos y observables; mientras que la segunda se refiere a una carencia de información confiable y homologable que posibilite el análisis comparado. A partir de lo anterior, se parte del supuesto de ambigüedad y de incertidumbre para entender el significado del concepto de apropiación de políticas públicas y, más aún, de su operacionalización en términos empíricos.

Esta investigación busca estimar el grado de apropiación de las políticas más allá del comportamiento de los indicadores. Como punto de partida, cabe señalar que existe una tendencia generalizada en el análisis social -y en correspondencia en la investigación educativa - por evaluar y comparar diferentes objetos de estudio para determinar cuáles están en las mejores y peores condiciones a partir de criterios de referencia. El presente trabajo busca desprenderse de la idea de clasificación como herramienta para la valoración del desempeño; esto se debe a que la política educativa tiene como población objetivo a todas las IES, independientemente de sus características. Dicho con otras palabras, los programas públicos asociables al ámbito educativo no están dirigidos únicamente a las instituciones más aptas; más aún, la aspiración formal de la acción gubernamental está orientada a la mejora del sistema educativo y no exclusivamente a empoderar a un pequeño grupo de instituciones.

Por lo anterior, hay que ser cautelosos sobre la interpretación de los índices propuestos. En principio, no miden desempeño sino que buscan de manera multidimensional estimar el grado en el que las instituciones han 
incorporado a su dinámica elementos asociables a los principales programas públicos; a ello denominaremos apropiación. De tal manera que esta aproximación posibilita un análisis basado en la combinación de la batería de programas que integran la política educativa. Esto pone en contraste la dinámica de implementación homogénea de políticas públicas con una realidad institucional diversa en términos de capacidades, contextos e historias.

Para el análisis se incluyen datos del 2014 de cuarenta IES que obtuvieron de la información que integra el Estudio Comparativo de las Universidades Públicas Mexicanas. La elección de estos casos se sustenta en lo que Ibarra (2001) denominó Sistema Universitario Mexicano (SUM) y en el Laboratorio de Análisis Institucional del Sistema Universitario Mexicano (LAISUM) mismas que para el año 2013 atendían aproximadamente al 43\% de la matrícula de nivel superior a nivel nacional. También se consideró su condición de universidades autónomas, en función de la personalidad jurídica que les otorga su Ley Orgánica. En la tabla 1 se muestran las instituciones analizadas.

Tabla 1.

Instituciones seleccionadas para el análisis.

\begin{tabular}{lll}
\hline Institución & Siglas & Matrícula 2013 (licenciatura) \\
\hline Benemérita Universidad Autónoma de Puebla & BUAP & 56,530 \\
\hline Instituto Politécnico Nacional & IPN & 101,212 \\
\hline Instituto Tecnológico de Sonora & ITSON & 15,737 \\
\hline Universidad Autónoma Agraria Antonio Narro & UAAA & 4,930 \\
\hline Universidad Autónoma Benito Juárez de Oaxaca & UABJO & 15,676 \\
\hline Universidad Autónoma Chapingo & CHAPINGO & 47,02 \\
\hline Universidad Autónoma de Aguascalientes & UAA & 13,311 \\
\hline Universidad Autónoma de Baja California & UABC & 55,068 \\
\hline Universidad Autónoma de Baja California Sur & UABCS & 5,102 \\
\hline Universidad Autónoma de Campeche & UACAM & 5,772 \\
\hline Universidad Autónoma de Chiapas & UNACH & 20,441 \\
\hline Universidad Autónoma de Chihuahua & UACH & 27,386 \\
\hline Universidad Autónoma de Ciudad Juárez & UACJ & 24,863 \\
\hline Universidad Autónoma de Coahuila & UACOH & 21,411 \\
\hline Universidad Autónoma de Guerrero & UAGRO & 25,356 \\
\hline Universidad Autónoma de la Ciudad de México & UACM & 12,797 \\
\hline Universidad Autónoma de Nayarit & UAN & 82,737 \\
\hline Universidad Autónoma de Nuevo León & UANL & \\
\hline & & UA14 \\
\hline
\end{tabular}




\begin{tabular}{|c|c|c|}
\hline Institución & Siglas & Matrícula 2013 (licenciatura) \\
\hline Universidad Autónoma de Querétaro & UAQRO & 17,300 \\
\hline Universidad Autónoma de San Luís Potosí & UASLP & 23,878 \\
\hline Universidad Autónoma de Sinaloa & UAS & 59,258 \\
\hline Universidad Autónoma de Tamaulipas & UAT & 34,613 \\
\hline Universidad Autónoma de Tlaxcala & UTLX & 12,505 \\
\hline Universidad Autónoma de Yucatán & UADY & 13,715 \\
\hline Universidad Autónoma de Zacatecas & UAZ & 19,400 \\
\hline Universidad Autónoma del Carmen & UACAR & 4,770 \\
\hline Universidad Autónoma del Estado de Hidalgo & UAEH & 23144 \\
\hline Universidad Autónoma del Estado de México & UAMEX & 45,161 \\
\hline Universidad Autónoma del Estado de Morelos & UAEMOR & 15,701 \\
\hline Universidad Autónoma Metropolitana & UAM & 50,928 \\
\hline Universidad de Colima & UCOL & 11,874 \\
\hline Universidad de Guadalajara & UDG & 96,534 \\
\hline Universidad de Guanajuato & UGTO & 17,045 \\
\hline Universidad de Quintana Roo & UQROO & 4,197 \\
\hline Universidad de Sonora & UNISON & 29,377 \\
\hline Universidad Juárez Autónoma de Tabasco & UJAT & 27,869 \\
\hline Universidad Juárez del Estado de Durango & UJED & 13,750 \\
\hline Universidad Michoacana de San Nicolás de Hidalgo & UMSNH & 38,980 \\
\hline Universidad Nacional Autónoma de México & UNAM & 190,336 \\
\hline Universidad Veracruzana & UV & 58,706 \\
\hline Total nacional UPM & 40 & $1,310,984$ \\
\hline Total nacional & & $3,309,221$ \\
\hline
\end{tabular}

Fuente: elaboración propia con base en el execum, disponible www.ecum.unam.mx, consultado el 10 de abril de 2015

\section{La construcción de indices multidimensionales como herramienta para entender el grado de apropiación de políticas públicas}

Para entender cómo las UPM se apropian de la política educativa es necesaria una aproximación empírica que combine factores objetivos, medibles y asociables a los programas públicos, así como una discusión cualitativa para entender cuál es el alcance del propio concepto de apropiación de políticas públicas. En este trabajo se presenta una propuesta de índices multidimensionales basados en conjuntos de lógica difusa para cuantificar en grado en el que las UPM alinean su dinámica a los escenarios planteados en los diferentes programas. 
Se discuten tres aspectos clave en el análisis basado en índices multidimensionales: 1) ambigüedad conceptual; 2) limitaciones empíricas en la calidad de la información; 3) restricciones de los propios índices.

Primero, en lo que respecta a la ambigüedad conceptual, particularmente en el estudio social, podemos destacar la falta de criterios que determinen los elementos que componen un fenómeno particular. Desde la perspectiva del ciclo de la política pública, los elementos mínimos que la integran son el problema público, su definición, su identificación y la selección de opciones, la implementación y la evaluación (Parsons, 2012). En ese sentido, se da por sentado que la población objetivo a la que está dirigida está implícitamente de acuerdo con las condiciones planteadas en las reglas de operación. Esto funciona bien en la política social dado que mayormente es de corte asistencialista y está planteada a grupos más o menos homogéneos.

Sin embargo, la política de educación superior parte de un supuesto distinto ya que el universo potencial de beneficiarios es diverso en factores asociables al contexto y a las capacidades institucionales. Esto da como resultado la ampliación de las brechas de desigualdad, ya que habrá universidades con mejores condiciones para atender los retos de la política, que en un sentido general podemos resumir en el aumento de matrícula, la diversificación de la oferta educativa, la mejora de la calidad y el desarrollo de infraestructura. Como consecuencia, la noción de apropiación conduce a ambigüedad e incertidumbre, ya que sería un error asumir que las instituciones implementan actividades similares obteniendo convergencia y homogeneidad en los resultados. Aquí se alude al segundo punto enunciado antes: la información asociada a este aspecto es limitada, pues en principio no están del todo claros cuáles son los indicadores deseables en términos de la política para determinar una adecuada apropiación... Por ejemplo, ¿cuál es la proporción ideal de profesores en el Sistema Nacional de Investigadores (SNI) o con perfil Programa de Mejoramiento de Profesorado (PROMEP)? Para efectos del presente escrito, la aproximación es a través de la integración de indicadores que se podrían asociar como resultado de la participación en la dinámica de la política educativa. Asimismo, al no contar con parámetros de referencia, los rangos se establecieron a partir de una realidad concreta basada en la propia distribución de las instituciones estudiadas.

Por último y en tercer lugar, la creación de índices multidimensionales tiene inherentemente una serie de restricciones. La más importante radica en el supuesto de una realidad social compleja explicada a partir de un grupo limitado de variables. Bajo esta lógica, se propone el uso de índices basados en conjuntos de lógica difusa dado que se busca una explicación integral de un fenómeno. A diferencia de los índices ponderados convencionales - también 
denominados sintéticos - , su diseño se soporta en tres elementos: a) conceptualización basada en conocimiento sustantivo, es decir, a través de la conjunción de teorías, conceptos, opiniones de expertos, tendencias y casos similares; b) comportamientos individuales explicados por funciones de pertenencia, lo cual posibilita grados de pertenencia diferenciados y correspondencia múltiple y simultánea entre clases; c) comportamiento basado en tablas de verdad. En otras palabras, son definidos a priori todos los posibles escenarios derivados de la combinación de las variables definidas (Salas y Flores, 2017; Salas y Flores 2016).

Por tanto, se construyeron índices multidimensionales a partir de variables reportadas por las propias universidades que pueden ser asociados directamente como resultados de los diferentes programas que integran la política educativa. En todos los casos se refieren al grado de presencia de las variables involucradas como una medida para estimar el grado de apropiación. Es decir, se cuantifica cómo la gestión institucional está alineada a las aspiraciones y los escenarios deseables planteados a nivel federal. Los índices se acotan a las funciones sustantivas de docencia y de investigación a través de dos índices con sus respectivos subíndices.

El primer índice denominado calidad en la docencia se compone por dos subíndices que integran elementos asociables a la evaluación externa por parte de los Comités Interinstitucionales para la Evaluación de la Educación Superior (CIEES), acreditación por los organismos reconocidos por el Consejo para la Acreditación de la Educación Superior (COPAES), el Programa Nacional de Posgrados de Calidad (PNPC) y el PROMEP, los cuales se muestran a continuación en conjunto con los modelos empleados (en el anexo 1 se muestran las funciones y tablas de verdad respectivas):

Figura 1.

Modelos empleados para el cálculo de los subíndices asociados al eje calidad en la docencia.

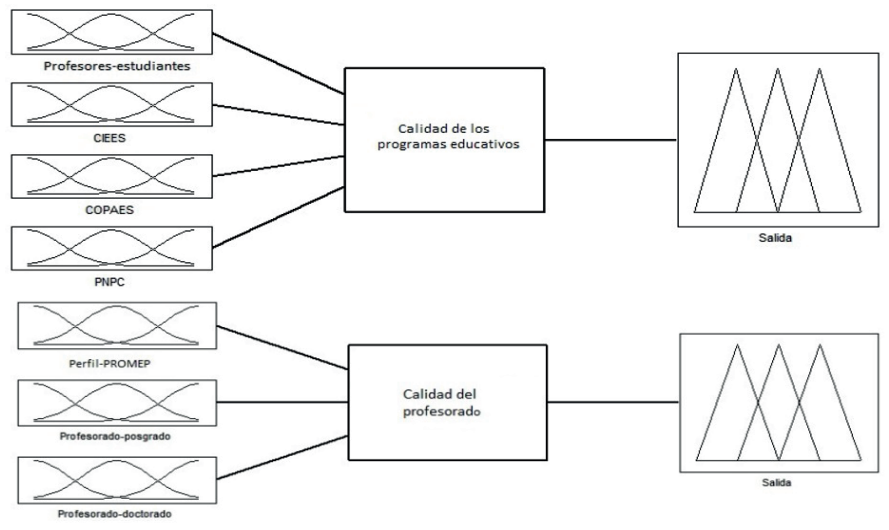

Fuente: elaboración propia. 
En lo que respecta a la investigación, se construyó un índice denominado Calidad de la investigación, el cual está conformado por tres subíndices que integran elementos asociables al PROMEP, PNPC, el SNI y el SCRMCYT, tal como se ilustra a continuación:

Figura 2.

Modelos empleados para el cálculo de los subíndices asociados al eje de calidad de la investigación.
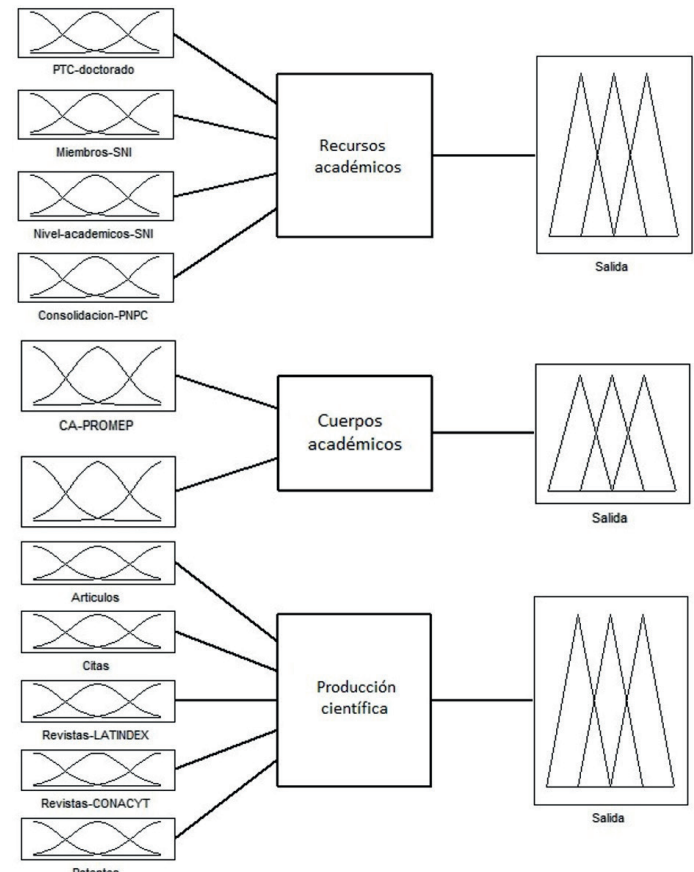

Fuente: elaboración propia.

\section{Análisis y discusión de los índices}

La interpretación de los índices propuestos se refiere al grado de apropiación de las políticas. Buscan estimar el nivel de cumplimiento de indicadores directamente atribuibles a la política educativa con la intención de hacer una valoración relativa a su grado de apropiación. Cabe destacar que en ningún sentido pretenden evaluar la eficiencia de las instituciones en el manejo de sus recursos o la pertinencia de sus mecanismos de gestión. Un valor resultante de cero representa que no están presentes indicadores asociados a los diferentes programas, mientras que una salida de uno refleja una total apropiación. Complementariamente, cabe destacar que la propia política educa- 
tiva no plantea valores deseables a los indicadores, de manera que las UPM maximizan sus vocaciones a partir de sus propios objetivos y capacidades institucionales.

En lo que respecta al eje de Calidad de la Docencia el índice propuesto está compuesto por dos subíndices que suponen distintos tipos de acción institucional para su apropiación. El primero - Calidad de los Programas Educativos - involucra directamente al proceso de enseñanza aprendizaje en aspectos tales como la pertinencia de planes y programas de estudio, la infraestructura, el equipamiento físico y tecnológico, los procesos académicos y administrativos, así como la habilitación del profesorado. En un sentido general reflejan al colectivo universitario y su atención se basa en las capacidades de la organización.

Por su parte, el segundo subíndice - Calidad del Profesorado - refleja el comportamiento individual de los profesores en términos de su grado de habilitación. En términos de gestión institucional se refiere mayormente al establecimiento de condiciones favorables e incentivos para que los profesores opten por fortalecer y evaluar sus perfiles que, a diferencia del primer subíndice, se basan en los sujetos. En la tabla 2 se muestran los resultados.

En lo que respecta al subíndice de Calidad de los Programas Académicos se identifican valores promedio menores con respecto al otro subíndice, sin embargo, la dispersión de datos es sustancialmente menor. Esto refleja en un sentido amplio la orientación de las universidades a cumplir con los parámetros derivados de la evaluación externa tanto en pregrado como en posgrado. Por tal razón es factible pensar que la cultura de la evaluación por instancias ajenas a las propias instituciones, iniciada durante la década de los noventa, ha sido asimilada de manera relativamente homogénea. Lo anterior tiene matices que harían pensar que dicha apropiación no necesariamente es sinónimo de mejora continua. Para ejemplificar, tanto los esquemas de evaluación de los ciees como los parámetros de acreditación de COPAES y PNPC han sufrido pocos cambios en términos del núcleo central de aspectos evaluados, y en el mejor de los casos han sido incrementales. Esto ha permitido que las UPM reduzcan las curvas de aprendizaje para presentar evidencia de sus procesos en los términos de las instancias evaluadoras. Esto podría en parte explicar el acelerado crecimiento de programas educativos evaluados y acreditados.

Desde esta perspectiva, la apropiación de la política cuenta con restricciones institucionales en términos del universo de la oferta educativa. Si bien se señaló que existe una adaptación organizacional en términos de la lógica de las evaluaciones externas, tampoco hay capacidad para suponer que la totalidad de los programas educativos estén acreditados. 
Por su parte, el subíndice de calidad del profesorado presenta valores promedio mayores evidenciando que esta vertiente ha contado con mejores condiciones para su apropiación. Desde esta lógica, la mayor profesionalización de las plantas académicas no se explica exclusivamente por la creación de incentivos institucionales aislados para incrementar los grados académicos y la diversificación de las actividades que realizan los profesores. Por el contrario, es factible pensar que como resultado de la asimilación de los escenarios planteados en los programas asociables a este rubro, las universidades adaptaron sus mecanismos de contratación con la finalidad de incrementar en el corto plazo sus indicadores.

Un posible efecto de lo anterior, es que los profesores, incorporados bajo esta lógica, contaban de origen con un perfil equilibrado de docencia, de gestión y de investigación, dando como resultado un incremento en algunos indicadores a un ritmo mayor que el que supone la formación en el posgrado de un profesor con licenciatura.

De manera integrada, el promedio de ambos subíndices muestra que las diferencias entre los casos analizados no son pronunciadas o claramente contrastantes. Esto hace suponer que las políticas para la educación superior lograron orientar la operación de las instituciones al margen de las visibles diferencias que existen entre ellas. Esto implica el establecimiento y la consolidación de una cultura de evaluación externa a diferentes niveles (planes y programas/plantas docentes, pregrado/posgrado), cuya existencia durante la década de 1980 era prácticamente inexistente.

En lo que respecta al índice de Calidad de la Investigación, está compuesto por tres subíndices que suponen aspectos complementarios asociables a esta función sustantiva. El primero - Recursos Académicos - valora el potencial con el que cuentan las universidades para el desarrollo de investigación, considerando tanto el nivel de habilitación de sus investigadores como de los programas de posgrado acreditados, el natural campo de acción.

El segundo subíndice - Cuerpos Académicos- busca cuantificar el grado de asociación de los académicos para actividades de investigación. A diferencia del subíndice anterior, su conformación no está reducida a un pequeño grupo de científicos sino que está abierta a un grupo más amplio que considera a los Profesores de Tiempo Completo (PTC). Refleja de manera puntual el impacto que ha tenido en la investigación la incorporación de nuevas funciones al perfil de los académicos, a través de los mecanismos que asociación para conciliar diferentes niveles de habilitación.

Finalmente el tercer subíndice - Producción Científica - refleja la capacidad de los investigadores para publicar los resultados de sus investigaciones. En alguna medida es resultado de los factores involucrados en el subíndice 
de recursos académicos. Los valores obtenidos presentan la mayor dispersión, haciendo evidente que la consolidación de cuadros de investigación de alto nivel trasciende a los mecanismos planteados en los programas públicos asociables, ya que se requieren de procesos más largos de maduración sin que ello garantice su incremento en el tiempo. Los resultados de eje de análisis se muestran en la tabla 3.

En cuanto al subíndice de Recursos Académicos se destaca que sus valores promedio reflejan la menor apropiación para este eje de análisis y son fiel reflejo de la baja proporción de capital humano de alto nivel en México. En otras palabras, el crecimiento de la comunidad científica reconocida por el CONACYT - investigadores y programas de posgrado - en términos relativos se ha incrementado de manera sostenida, aunque en valores absolutos sigue representado a una pequeña elite. Una de las implicaciones de lo anterior refuerza el concepto de ambigüedad en lo que respecta al alcance de los programas, pues no está claro cuál es el escenario ideal al que deben aspirar las universidades; en el mejor de los casos se crean rankings a partir de valores absolutos para clasificar a las UPM, privilegiando la diferenciación e implícitamente contribuyendo a favorecer las brechas de desigualdad.

El segundo subíndice - Cuerpos Académicos - tiene valores promedio mayores y equiparables al subíndice de producción científica. Sin embargo, una caracterísica que destaca es que presenta la menor dispersión. Esto significa que de los tres subíndices planteados, éste representa en términos del presente escrito la mayor homogeneidad en la apropiación. La lógica de lo anterior está en función del alcance de programa asociado a los CA de promep, que por su diseño es capaz de integrar las comunidades académicas considerando la gama de niveles de habilitación del profesorado. Asimismo, en términos de acción institucional se asocia mayormente al corto plazo, puesto que buscan incentivar el trabajo colaborativo a partir de la afinidad de intereses.

El tercer subíndice - Producción Científica - , como se señaló, cuenta con valores promedio al subíndice de Cuerpos Académicos, aunque presenta la mayor dispersión. Es decir, que este aspecto muestra en mayor medida las brechas de apropiación en aspectos claramente asociables a perfiles de alto nivel, y se asocia a productividad, citas y patentes. El resultado es que pocas instituciones concentran la mayoría de la producción científica, mientras que el resto lo hace de manera marginal haciendo evidente otra dimensión de la desigualdad.

En términos agregados, el índice de calidad de la investigación muestra claras diferencias entre instituciones, lo cual evidencia que la conformación de recursos humanos de alto nivel no sólo es producto de apoyos e incenti- 
vos sino de la consolidación misma de las comunidades científicas. Por lo que el grado de apropiación de estos programas está relacionado directamente con las características de la planta académica de cada universidad y, a diferencia del índice de calidad de la docencia, no parece factible una convergencia de valores en los próximos años. Por el contrario, pareciera existir una tendencia a una mayor diferenciación debido a la sinergia que genera la concentración de investigadores en algunas instituciones.

En este punto es evidente la ambigüedad de las políticas para delimitar los escenarios ideales a los que pueden acceder las UPM a partir de sus características. De manera que al discutir sobre la apropiación de la política no la podemos disociar de las restricciones dadas por las propias capacidades instituciones y sus alcances a través de vocaciones y objetivos institucionales. Para entender lo anterior, en la figura 3 se muestra el cruce de los ejes de Calidad de la Docencia y Calidad de la Investigación.

Figura 3.

Grado de apropiación de la PE en términos de los ejes de calidad de la docencia y calidad de la investigación.

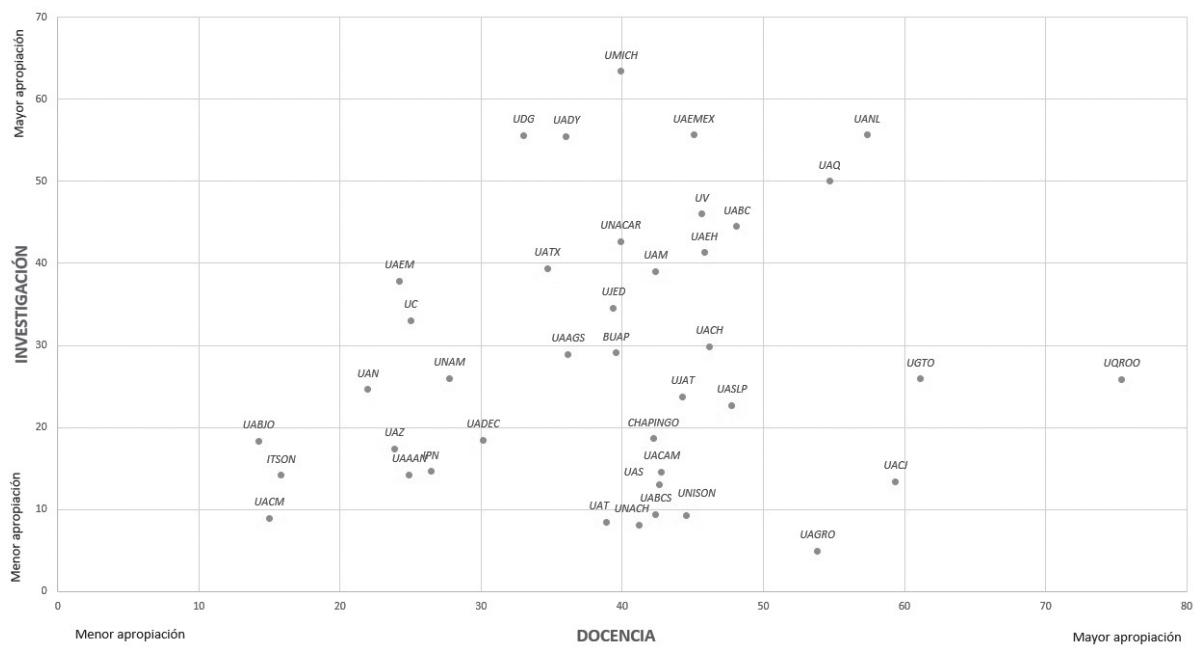

Fuente: elaboración propia.

Como se ilustra, existe una gran dispersión entre las instituciones analizadas. Se destaca que no hay alguna institución que equilibradamente presente altos niveles de apropiación en elementos asociables a la docencia y la investigación. Una posible explicación de lo anterior está compuesta por factores endógenos y exógenos a las propias universidades. 
Con respecto al peso atribuible a las instituciones, es evidente que tienen capacidades limitadas. En el mejor de los casos tienen vocaciones hacia ciertos aspectos específicos tales como mayor apoyo a los investigadores en el SNI, un énfasis institucional a contar con un mayor número de programas de licenciatura acreditados, una mayor oferta de posgrados en el marco del PNPC, o cualquier otra actividad, las cuales están reflejadas en mayor o menor medida en los programas de desarrollo institucional. Asimismo, este tipo de aproximaciones hacen evidente que las fortalezas de ciertas áreas del conocimiento o dependencias diferencian a las universidades en términos relativos. En otras palabras, la institución líder en el ranking de alguna variable, posiblemente cuente con una comunidad académica más grande matizando el impacto absoluto de la apropiación de la política.

En términos organizacionales también se generan niveles asimétricos de profesionalización, por ejemplo, se crean áreas nuevas con su propia estructura jerárquica para la atención de un programa en particular, mientras que otros se gestionan indistintamente mezclados por personal poco especializado en las reglas de operación de los distintos programas de políticas y de requisitos para su participación. Asimismo y hasta cierto punto, la participación institucional es optativa y está en función de las aspiraciones institucionales, pues la mayor parte del financiamiento proviene de fondos irreductibles y sólo una fracción procede de los denominados fondos concursables.

Por su parte, la componente asociable a la PE parte de la oferta de una amplia gama de programas enfocados a diferentes aspectos, tales como la calidad de los programas educativos, la ampliación de la oferta, los apoyos para la manutención de los estudiantes, la diversificación de la oferta educativa y el mejoramiento de la infraestructura, entre otros. Sin embargo, lo limitado de los recursos destinados a su operación crea un escenario de competencia entre instituciones para obtener financiamiento para su implementación. Esto genera simultáneamente círculos virtuosos y viciosos. Por una parte, habrá un grupo de instituciones que se apropien con mejores condiciones a las reglas de programas específicos haciéndolos exitosos en términos de sus indicadores, mientras que, por otro lado, existirá otro grupo marginado de estos apoyos puesto que no cuenta con las condiciones mínimas para competir.

Complementariamente, el propio diseño de los programas genera incertidumbre en términos absolutos, ya que en el mejor de los casos valida - mayormente en aspectos financieros - a las instituciones desde su propia lógica. Por ejemplo, si una UPM determina crear un nuevo laboratorio con un fondo de infraestructura, el cumplimiento está en función de su construcción y su puesta en marcha y no en las necesidades de una región, el grado de replicabilidad en otro contexto o el uso compartido con otras 
instituciones para fortalecer al sistema educativo mismo. Es decir, fomenta esfuerzos aislados justificados mediante la propia capacidad de gestión de las instituciones.

Bajo esta lógica, la combinación de autonomía - en la gestión - universitaria y una amplia gama de programas públicos atribuible a la política educativa de nivel superior, generan fuertes asimetrías entre y al interior de las instituciones, así como ambigüedad sobre cuáles son los indicadores y estándares a los que deben aspirar las universidades. Como prueba de lo anterior, los propios informes de actividades de los rectores enfatizan en los principales logros de cada universidad sin que necesariamente estén homologados. Esto hace prácticamente imposible una comparación compleja sobre las convergencias y asimetrías del subsistema educativo superior reduciendo el análisis a la clasificación por indicadores individuales para establecer rankings, lo cual orienta a las UPM a un modelo de competencia, a pesar de que la PE está planteada para todo el sistema.

\section{Conclusiones}

Derivado del análisis multidimensional mostrado, nos aproximamos empíricamente para entender la apropiación de la política educativa en el SUM. En principio se evidenció la ambigüedad de sus propósitos en dos niveles: por programa y en lo colectivo. En cuanto al programa se demuestra empíricamente la existencia de incertidumbre en términos operativos, dado que no existen parámetros de referencia a los que las universidades, de acuerdo a sus propias características, deban aspirar. Parece ser que la configuración actual incentiva por una parte la competencia por los recursos limitados, mientras que por la otra promueve la autovalidación del impacto de los recursos públicos por parte de las universidades, ya que no está considerado el desarrollo equilibrado de este subsistema educativo.

Por otra parte y al margen de los puntos de partida distintos, se observa de manera relativamente homogénea una orientación hacia el cumplimiento de indicadores, posiblemente explicado por el financiamiento asociado. Sin embargo, la transformación interna que se generó no tiene marcha atrás, puesto que las instituciones han integrado, como parte de sus actividades, la evaluación externa y la dinámica financiera asociada. Esto repercute en la propia definición de los objetivos institucionales; en la planeación; en las adecuaciones a la estructura jerárquica; en los mecanismos de contratación, de creación y de actualización de la oferta educativa; en el desarrollo de perfiles docentes más integrales; en la definición de nuevos procesos; en la adaptación de la normativa; en el desarrollo de plataformas para la transparencia. 
En cuanto al análisis colectivo de los diferentes programas, los resultados obtenidos dejan entrever implicaciones todavía mayores. Las UPM cuentan con un conjunto limitado de capacidades, las cuales se relacionan, entre otros elementos, con su historia, su contexto, sus objetivos institucionales, sus capitales académico y político. Ello conduce a que las instituciones se concentren en ciertos programas ante la insuficiencia práctica para atender todas las dimensiones de la política educativa. Si agregamos la componente de estabilidad en el tiempo de los diferentes programas, podemos pensar en un grado de especialización organizacional que en términos agregados permite identificar la vocación de cada institución. Prueba de lo anterior es la profesionalización de gestores y la creación de áreas funcionales para atender programas públicos específicos.

La metodología multidimensional utilizada no busca aislar las variables clave, por el contrario, pretende obtener un panorama general del fenómeno. Como resultado, la apropiación de la política sustancialmente conduce a la ampliación de las brechas de inequidad puesto que este proceso no es homogéneo y constante, sino más bien parcial y asimétrico, por lo que las instituciones con mayores capacidades serán las grandes ganadoras. Sin embargo, no está del todo claro cuál es el piso mínimo al que las universidades deben aspirar para contribuir a mejorar el subsistema de educación superior, ya que no hay que omitir que la población objetivo de la política no se reduce al grupo de las instituciones más aptas. Por el contrario, con la configuración actual se corre el riesgo de fomentar cada vez más la diferenciación.

\section{Referencias}

Aguilar, Luis Fernando (1993). La implementación de las políticas. México D. F.: Miguel Ángel Porrúa.

Badillo, Rosalba; Buendía, Angélica y Krucken, Georg (2015). Liderazgo de los rectores frente a la "tercera misión" de la universidad: Visiones globales, miradas locales. Revista Mexicana de Investigación Educativa, 20(65), 393-417.

Birbaum, Robert (1983). Maintaining Diversity in Higher Education. San Francisco: Jossey-Bass.

Birnbaum, Robert (1983). Maintaining Diversity in Higher Education. San Francisco: Jossey-Bass.

Buendía, Angélica (2013). Genealogía de la evaluación y acreditación de instituciones en México. Perfiles educativos, 35, 17-32.

Buendía, Angélica (2014). Evaluación y acreditación de programas académicos en México: revisar los discursos, valorar los efectos: el caso de cinco universidades públicas mexicanas. México D. F.: Asociación Nacional de Universidades e Instituciones de Educación Superior. 
Buendía, Angélica; García, Susana; Grediaga, Rocío; Landesman, Monique; Rodríguez, Roberto; Rondero, Norma; Rueda, Mario y Héctor Vera (2017). Queríamos evaluar y terminamos contando: alternativas para la evaluación del trabajo académico. Perfiles Educativos, 39(157), 200-220.

Capano, Giliberto (2011). Government Continues To Do Its Job. A Comparative Study of Governance Shifts in the Higher Education Sector. Public Administration, 89(4), 1622-1642.

Cerna, Lucie (2013). The Nature of Policy Change and Implementation: A Review of Different Theoretical Approaches. París: Organización para la Cooperación y el Desarrollo Económicos.

Chehaibar, Lourdes; Díaz Ángel y Mendoza, Javier (2007). Los Programas Integrales de Fortalecimiento Institucional. Apuntes para una evaluación desde las universidades. Perfiles Educativos, 29(117), 41-67.

Clark, Burton (1986). The higher education system: Academic organization in cross-national perspective. Berkeley: University of California Press.

Cohen, Michael y March, James (1974). Leadership and ambiguity: The American college president. Boston: Harvard Business School Press.

Díaz, Ángel (2008). Impacto de la evaluación en la educación superior mexicana. Un estudio en las universidades públicas estatales. México D. F.: Plaza y Valdés.

Flores, Lucio y Luhrs, Margarita (2013). Evaluación de programas públicos en el marco de la realidad social. Metodología basada en la lógica difusa como instrumento para el análisis de fenómenos sociales. Revista Latinoamericana de Metodología de la Investigación Social, (5), 8-23.

Frank, David y Meyer, John (2007). University expansion and the knowledge society. Theory and Society, 36(4), 287-311.

Fresán, Magdalena y Taborga, Huáscar (2000). Tipología de instituciones de educación superior. México D. F.: Asociación Nacional de Universidades e Instituciones de Educación Superior.

García, María Luisa; Flores, Lucio y Venegas, Beatriz Adriana (2016). Análisis del desarrollo sostenible en espacios locales. Aplicación de la teoría de conjuntos difusos. Íconos. Revista de Ciencias Sociales, (54), 173-197.

Huisman, Jeroen; Meek, Lynn y Wood, Fiona (2007). Institutional Diversity in Higher Education: a Cross-National and Longitudinal Analysis. Higher Education Quaterly, 61(4), 563-577.

Ibarra, Eduardo (2001). La Universidad de México hoy: gubernamentalidad y modernización. México D. F.: Universidad Nacional Autónoma de México / Universidad Autónoma Metropolitana / Asociación Nacional de Universidades e Instituciones de Educación Superior.

Ibarra, Eduardo y Buendía, Angélica (2013). Compendio del Sistema Universitario Mexicano: Perfil institucional, datos e indicadores. México D. F.: Universidad Autónoma Metropolitana - Unidad Xochimilco.

McLaughlin, Milbrey (1987). Learning from experience: lessons from policy implementation. Education Evaluation and Policy Analysis, 9(2), 171-178.

Meek, Lynn; Goedegebuure, Leo y Huisman, Jeroen (2000). Understanding diversity and differentiation in higher education: an overview. Higher Education Policy, (13), 1-6. 
Montaño, L. (2012). Los enredos de la evaluación. Una perspectiva institucionalista acerca del desempeño académico. En Barba, Antonio y Lobato, Odette (Coords.), Instituciones de educación superior, políticas públicas y organización. México D. F: Miguel Ángel Porrúa.

Neave, Guy (2000). Diversity, differentiation and the market: the debate we never had but which we ought to have done. Higher Education Policy, (13), 7-22.

Parsons, Wayne (2012). Políticas Públicas. Una introducción a la teoría y la práctica del análisis de políticas públicas (segunda reimpresión). México D. F.: Facultad Latinoamericana de Ciencias Sociales, sede México.

Ragin, Charles (2014). The Comparative Method. Moving beyond qualitative and quantitative strategies. Oakland: University of California Press.

Ragin, Charles (2006). The limitations of net-effect thinking. En Rihoux, Benoit y Grimm, Heike (Ed.), Innovative comparative methods for policy analysis. Nueva York: Springer.

Rodríguez, Roberto (2017). Más autonomía o más gobernanza. Un dilema universitario. Revista Mexicana de Investigación Educativa, 75(22), 1015-1020.

Salas, Iván Alejandro y Flores, Lucio (2017). Aplicación de conjuntos difusos para crear índices multidimensionales. Una aproximación para cuantificar la calidad del empleo. Acta Universitaria, 27(2), 65-82.

Salas, Iván Alejandro y Flores, Lucio (2016). Modelado de conjuntos difusos para cuantificar la calidad del empleo. Una aproximación conceptual. Revista Ciencia y Universidad, (34), 7-36.

sep. (2016). Evaluación de Diseño. Fortalecimiento de la Calidad Educativa. Instituto de Investigaciones Dr. José María Luis Mora.

Stadtman, Verne (1980). Academic adaptations: higher education prepares for the 1980s and the 1990s. San Francisco: Jossey Bass.

Teicher, Ulrich (2008). Diversification? Trends and explanations of the shape and size of higher education. Higher education, (56), 349-379.

Trow, Martin (1995). Diversity in higher education in the United States of America. CVCP Seminar on Diversity in Higher Education. Londres.

Trow, Clark (1970). An educational model for social learning. Psychology in the Schools, 7(3), 237-240.

Varone, Frederic, Rihoux, Benoit y Marx, Axel (2006). A new method for policy evaluation? Longstanding challenges and the possibilities of Qualitative Comparative Analysis (qca). En Rihoux, Benoit y Grimm, Heike (Ed.), Innovative comparative methods for policy analysis. Nueva York: Springer.

Vught, Frans (2007). Diversity and Differentiation in Higher Education Systems. En Vught, Frans (Ed.), Mapping the Higher Education Landscape. Towards a European Classification of Higher Education. Bruselas: Springer.

Weick, Karl (1976). Educational Organizations as Loosely Coupled Systems. Administrative Science Quarterly, 21(1), 1-19.

Yamasaki, Sakura y Spreitzer, Astrid (2006). Beyond methodological tenets. The worlds of qca and SNA and their benefits to policy analysis. En Rihoux, Benoit y Grimm, Heike (Ed.), Innovative comparative methods for policy analysis. Nueva York: Springer.

Zha, Qiang (2009). Diversification or homogenization in Higher Education: A Global Allomorphism Perspective. Higher Education in Europe, 34(3-4), 459-479. 


\section{Anexos}

\section{Anexo 1.}

Funciones y tablas de verdad asociados al índice de calidad en la docencia.

\subsection{Calidad de los programas educativos.}
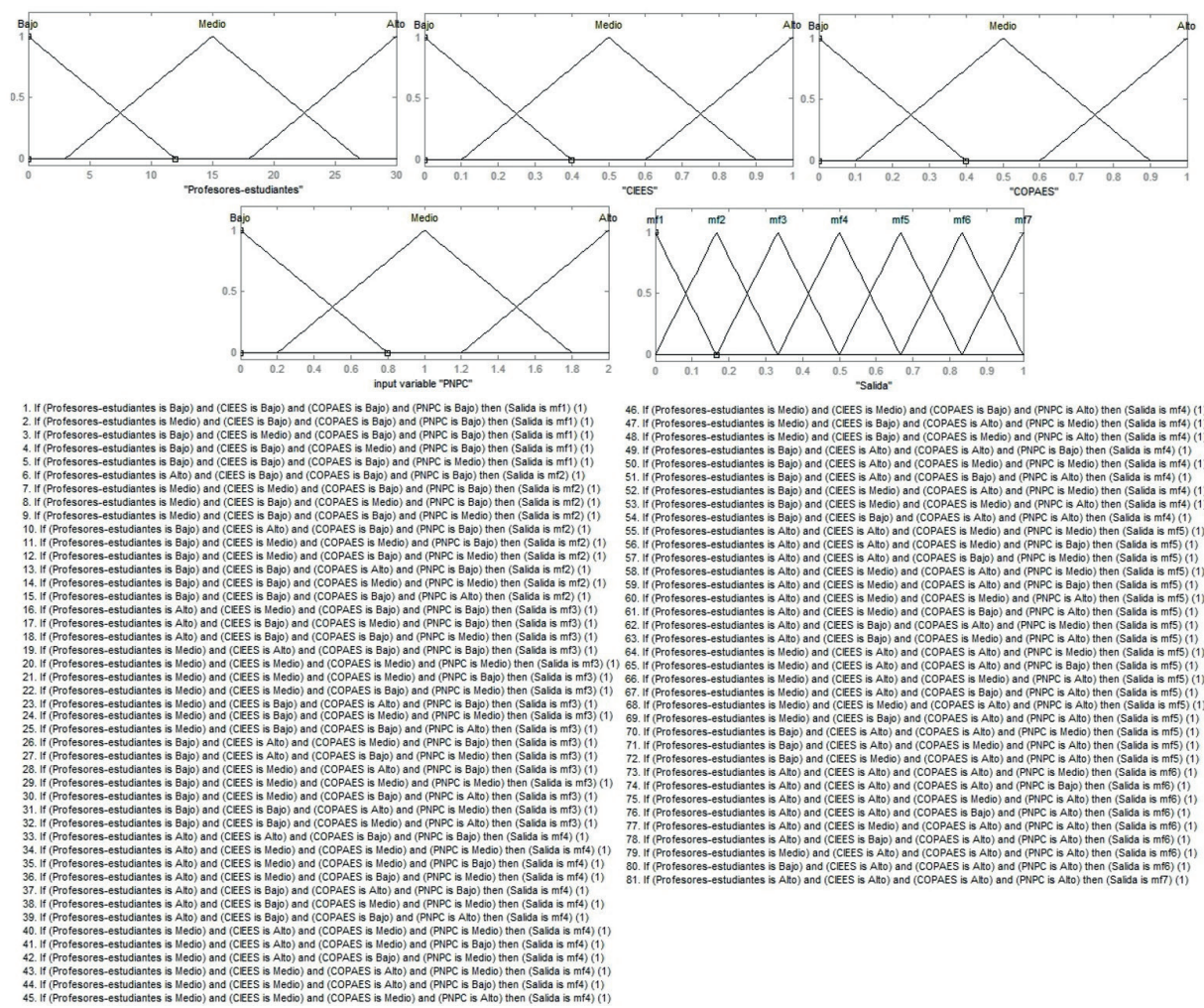

Fuente: elaboración propia.

\subsection{Calidad del profesorado}
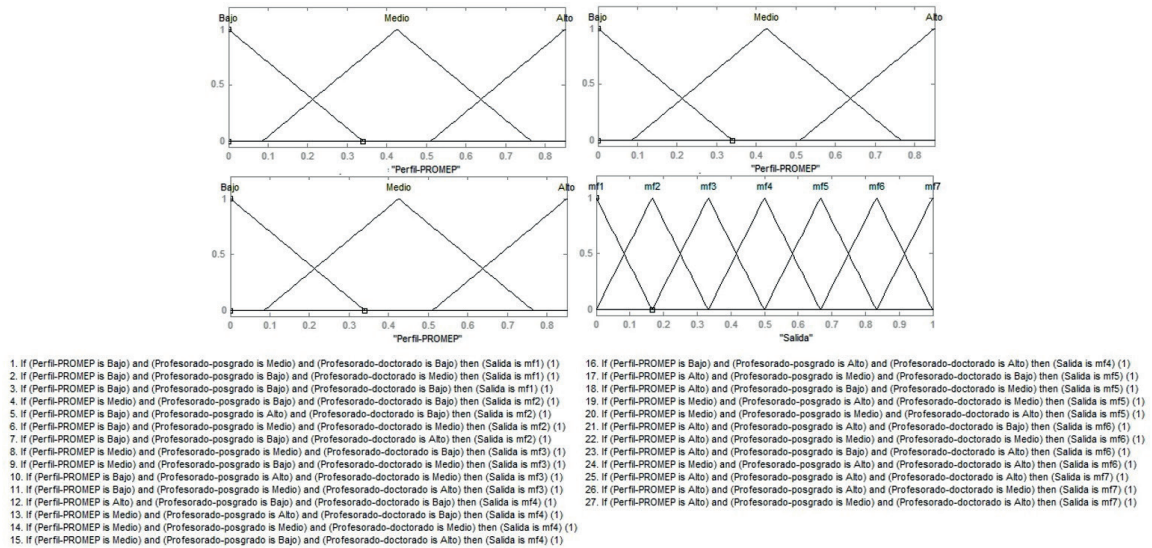

Fuente: elaboración propia. 
Anexo 2.

Funciones y tablas de verdad asociados al índice de calidad de la investigación.

\subsection{Recursos académicos.}
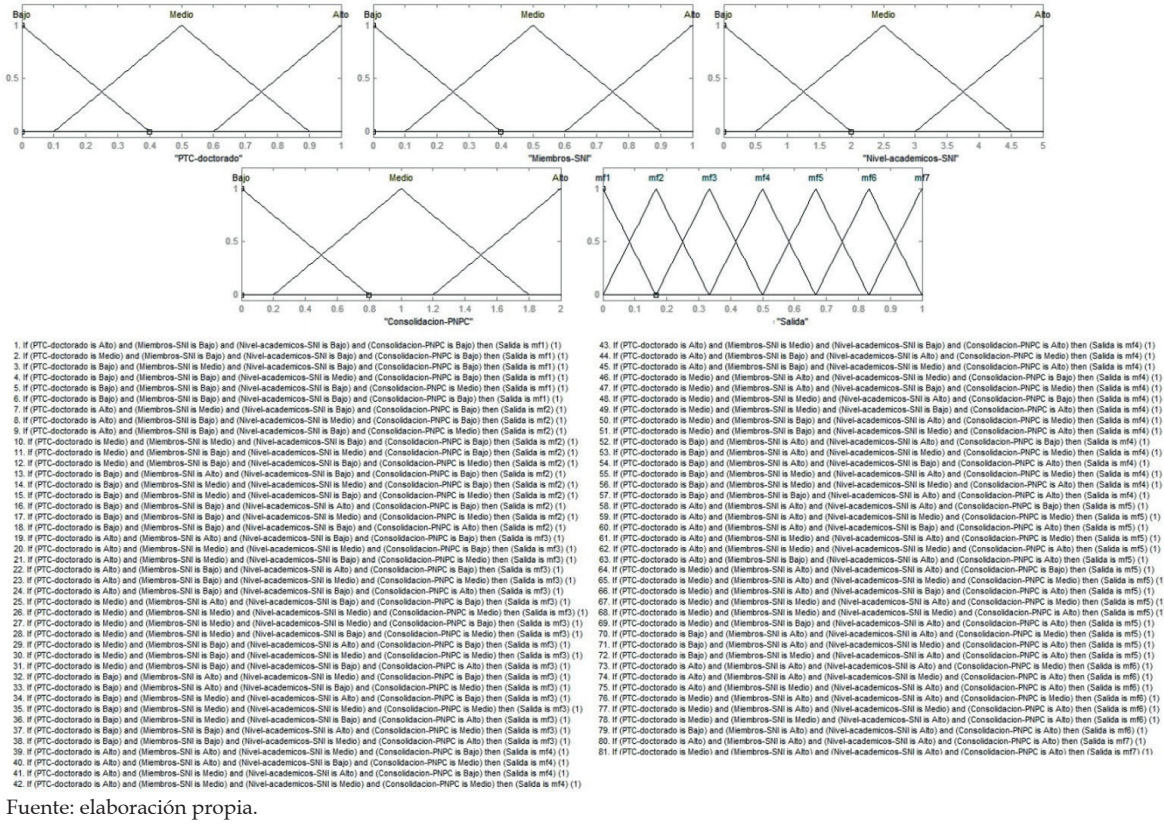

Fuente: elaboración propia.

\subsection{Cuerpos académicos.}
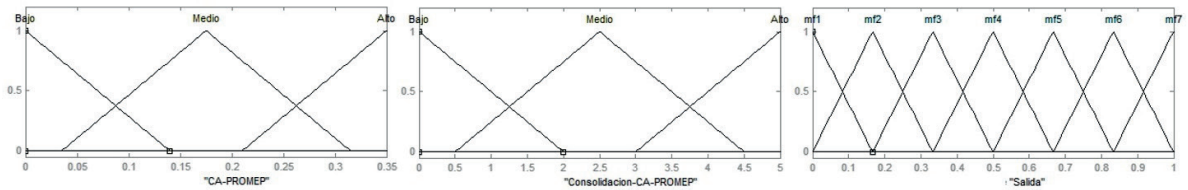

1. If (CA-PROMEP is Alto) and (Consolidacion-CA-PROMEP is Afto) then (Salida is $\mathrm{mF}$ ) (1)

2. If (CA-PROMEP is Atto) and (Consolidacion-CA-PROMEP is Medio) then (Salida is $m$ f5) (1)

3. If (CA-PROMEP is Atto) and (Consolidacion-CA-PROMEP is Bajo) then (Salida is mf4) (1)
4. If (CA-PROMEP is Medio) and (COnsolidacion-CA-PROMEP is Atto) then (Salida is mf5) (1)

4. If (CA-PROMEP is Medio) and (COnsolidacion-CA-PROMEP is Alto) then (Salida is mf5) (1)
5. If (CA-PROMEP is Medio) and (Consolidacion-CA-PROMEP is Medio) then (Salida is mf3) (1)

6. If (CA-PROMEP is Medio) and (Consolidacion-CA-PROMEP is Bajo) then (Salida is $\mathrm{m}$ (2) (1)

7. If (CA-PROMEP is Bajo) and (Consolidacion-CA-PROMEP is Aito) then (Salida is $\mathrm{m}+5$ ) (1)

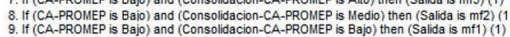

Fuente: elaboración propia.

2.3 Producción científica.
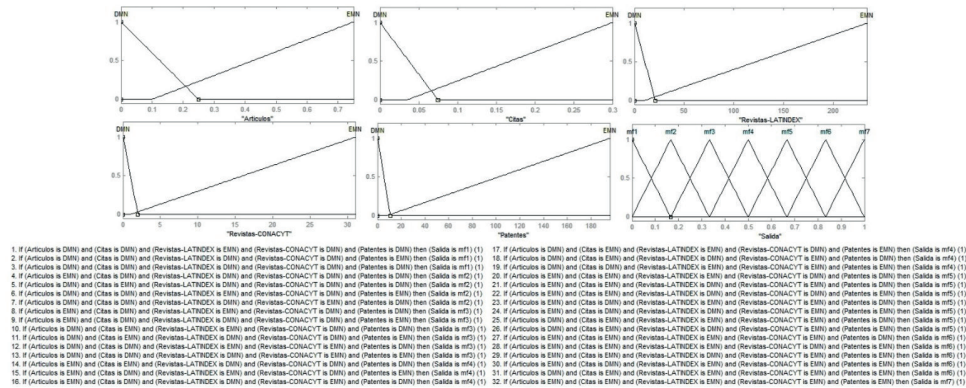

Fuente: elaboración propia. 
